\title{
Multicompetencia lingüística y cambio de código en el humor infantil
}

\author{
Susana Pastor Cesteros \\ Universidad de Alicante
}

\begin{abstract}
Los fenómenos de multilingüismo y multicompetencia lingüística han sido abordados desde muy diferentes perspectivas (Martin-Jones, Blackledge y Creese 2012; Cook y Wei 2016). El objetivo de este artículo, sin embargo, es ponerlos en relación con los procesos de cambio de código y con la percepción de la diversidad lingüística para estudiar su presencia y efecto en un corpus de 184 narraciones escritas humorísticas de niños de 8 a 12 años, obtenido en el marco del proyecto I+D La formación de la conciencia figurativa en la etapa de formación primaria: el humor y la fraseología (FFI2016-76047-P). El análisis cuantitativo y cualitativo del corpus ha permitido ver en las conclusiones hasta qué punto el conocimiento implícito y explícito de varias lenguas a esas edades incide en el cambio de código y en las referencias a otras lenguas y si ello aparece o no de un modo intencionado para provocar un efecto cómico (Timofeeva-Timofeev 2017).
\end{abstract}

Palabras clave: multicompetencia lingüística, cambio de código, humor infantil

\section{Introducción}

En el marco de los estudios sobre lingüística aplicada y plurilingüismo, el presente artículo tiene como objetivo analizar las muestras de multicompetencia lingüística y los cambios de código en un corpus de narraciones humorísticas de niños y niñas de entre 8 y 12 años. ${ }^{1}$ Pretendemos averiguar hasta qué punto el conocimiento de varias lenguas a esa edad incide en el cambio de código en dichos textos

1. Se trata del corpus CHILDHUM, creado a partir del proyecto de investigación Metapragmática del humor infantil: adquisición, perspectiva de género y aplicaciones (GRE14-19, Universidad de Alicante), dirigido por Larissa Timofeeva-Timofeev. Este corpus está siendo ampliado en el actual proyecto I+D La formación de la conciencia figurativa en la etapa de formación primaria: el humor y la fraseología (FFI2016-76047-P).

https://doi.org/10.1075/sic.00068.pas

Spanish in Context 18:1 (2021), pp. 56-82. issn 1571-0718 | e-issn 1571-0726

(c) John Benjamins Publishing Company

\section{2nd proofs}


humorísticos escritos y si dicho cambio aparece o no de un modo intencionado para provocar un efecto cómico. Paralelamente, las referencias a diversas lenguas y a su proceso de aprendizaje nos permitirán observar la actitud de los autores de los textos hacia el multilingüismo.

Con tal fin, presentamos a continuación en esta misma introducción el marco teórico y estado de la cuestión, tanto desde los estudios sobre multilingüismo como desde la metapragmática del humor infantil. En el segundo apartado, mostramos la metodología empleada para el diseño de la investigación (la recopilación del corpus, la identificación de los datos y su tratamiento). Posteriormente, analizamos y comentamos los resultados obtenidos, mostrando numerosos ejemplos, para finalizar con las conclusiones a las que hemos llegado a la luz de los mismos.

Desde un punto de vista teórico, el fenómeno de la multicompetencia lingüística, por su heterogeneidad y carácter pluridimensional, puede ser abordado desde muy diversas perspectivas. Comenzaremos por definir los conceptos claves. Dos de los que a menudo aparecen interrelacionados, pero que conviene distinguir desde un principio, son los de plurilingüismo y multilingüismo, y directamente relacionado con ellos, el de cambio de código. Veamos a continuación cómo define los dos primeros el Diccionario de términos clave de ELE (Martín Peris 2008): ${ }^{2}$

El término plurilingüismo hace referencia a la presencia simultánea de dos o más lenguas en la competencia comunicativa de un individuo y a la interrelación que se establece entre ellas. Los conocimientos y experiencias lingüísticas de un individuo pueden adquirirse bien en sus entornos culturales o bien en la escuela; se organizan en sistemas que se relacionan entre sí e interactúan, contribuyendo así a desarrollar la competencia comunicativa del sujeto. El multilingüismo, en cambio, es el conocimiento de varias lenguas o la coexistencia de distintas lenguas en una sociedad determinada. El multilingüismo puede lograrse, por ejemplo, diversificando la oferta de lenguas en un centro escolar o en un sistema educativo concreto. En la actualidad, el dominio de una, dos o más lenguas consideradas de forma aislada, deja de ser el objetivo de aprendizaje para dar paso a la consecución del plurilingüismo. [...] Las propuestas curriculares han incorporado recientemente una referencia explícita a la sensibilización de los aprendientes hacia la diversidad lingǘstica y cultural y el respeto a diferentes maneras de expresarse y actuar. [...] La competencia plurilingüe varía de acuerdo con las experiencias lingüísticas que haya vivido el individuo. [...] El concepto de competencia parcial en una lengua, lejos de significar un desarrollo imperfecto o pobre, es parte de una competencia plurilingüe que resulta enriquecida.

2. https://cvc.cervantes.es/ensenanza/biblioteca_ele/diccio_ele/diccionario/plurilinguismo .htm.

\section{2nd proofs}


Así pues, cuando hablamos de competencia plurilingüe nos referimos a que la competencia comunicativa de cualquier persona se conforma no solo a través de los conocimientos lingüísticos de su primera lengua (L1), sino también de los del resto de lenguas adicionales o segundas lenguas (L2), por las experiencias vividas con y a través de ellas. Desde un punto de vista cognitivo, todas ellas interactúan y se interrelacionan en diversa medida. Por ello, en cualquier acontecimiento comunicativo, un individuo puede recurrir a ellas y mostrar de ese modo, consciente o inconscientemente, su bagaje lingüístico. Ello se muestra, entre otros procedimientos, a través del 'cambio de código' (codeswitching en inglés, también traducido como 'alternancia lingüística'), definido en el ya citado Diccionario de términos clave de ELE como: ${ }^{3}$

el empleo alternativo de dos (o más) lenguas o dialectos en un discurso. Dicha alternancia es un fenómeno natural y común entre individuos bilingües, cuya competencia pragmática les permite escoger (incluso de un modo inconsciente) entre uno u otro código, según el interlocutor, la situación, el tema o el propósito de la interacción. [...] La elección de una u otra lengua como base puede responder tanto a razones psicolingüísticas como a razones sociolingüísticas.

Así pues, para que haya cambio de código ha de existir una previa competencia plurilingüe, que viene a equivaler a lo que autores como Cook han definido como multicompetencia lingüistica: "the knowledge of more than one language in the same mind or the same community. [...] everything a single person or a single community knows about all the languages they uses" (Cook 2012, 3768). Antes de seguir adentrándonos en este concepto, veamos de qué manera ha sido también enfocado desde los estudios sobre multilingüismo, en la medida en que este se constituye como un fenómeno multidimensional y holístico.

En efecto, y yendo de lo más general a lo más particular, el multilingüismo ha sido estudiado desde la sociolingüística como un acontecimiento social, con importantes implicaciones ideológicas, políticas y educativas, pero sin dejar de lado esta dimensión psicolingüística de 'la mente plurilingüe' a la que nos hemos referido (Moreno Cabrera 2016, 17-48). Existe a su vez abundante bibliografía sobre las directrices que habría de seguir una educación plurilingüe; en nuestro contexto más cercano, se ha trabajado esta cuestión especialmente desde realidades sociales bilingües con una larga tradición de estudios sociolingüísticos, como el País Vasco o Cataluña (Garcia-Azkoaga e Idiazabal 2015; Cenoz y Gorter 2015) y en relación directa con el fenómeno del translanguage (Cenoz 2017). El multilingüismo desde el punto de vista de la adquisición bilingüe del lenguaje infan-

3. https://cvc.cervantes.es/ensenanza/biblioteca_ele/diccio_ele/diccionario

/alternanciacodigo.htm

\section{2nd proofs}


til ha sido estudiado, entre otros, por Lleó (2006) y por Bronckart y Bronckart (2015). Más recientemente, su papel en el aprendizaje de una L2 ha sido también abordado por Ruiz de Zarobe y Ruiz de Zarobe (2017, 1-12). Ahora bien, probablemente una de las visiones más completas sobre todos estos y otros muchos aspectos podemos encontrarla de manera conjunta en The Routledge Handbook of Multilingualism (Martin-Jones, Blackledge y Creese 2012), que dedica sendos capítulos a los discursos sobre multilingüismo en distintos contextos históricopolíticos, al multilingüismo en la educación y en otros espacios institucionales, pero también a esta otra dimensión más personal, de realidades plurilingües vividas, que es la que en este artículo nos interesa. Nos referimos en particular a los capítulos sobre literacidad multilingüe (Warriner 2012) y sobre la relación del multilingüismo con las emociones (Pavlenko 2012). De este último destacamos la apreciación de que las emociones (por tanto, también indirectamente el humor) se manifiestan de un modo muy distinto en una L1 y en cualquier L2. Pavlenko explica cómo se establece esta relación en la adquisición del lenguaje infantil:

Two inter-related processes take place during primary language acquisition. The first is conceptual development, where children form emotion categories that include information from all modalities - visual, auditory, olfactory, tactile, kinesthetic, and visceral- and are subject to ongoing modification that take place in the language socialization process. The parallel process involves affective linguistic conditioning, where words and phrases acquire affective connotations and personal meanings through association and integration with emotionally charged memories and experiences [...]. Together, the two processes contribute to the perception of language embodiment, whereby first language (L1) words invoke sensory images, physiological reactions, and sometimes also feelings of anxiety, shame, or guilt. Foreign language words, on the other hand, do not elicit emotional reactions.

(Pavlenko 2012, 456)

Paralelamente, el factor edad es esencial, puesto que en este artículo trabajamos con informantes de entre 8 y 12 años. En ese sentido, destacamos el estudio sobre la conciencia pragmática y las actitudes lingüísticas de jóvenes aprendices realizado por Portolés $(2015,41-76)$, en particular porque fue llevado a cabo dentro del contexto valenciano, de donde procede también el corpus analizado en este artículo. Estas son algunas de las conclusiones a las que llega la autora sobre la actitud de sus informantes hacia las lenguas con las que entran en contacto en su realidad educativa:

... Not all language systems are equally valued. Our respondents have shown positive attitudes towards multilingualism. However, participants have reported the most positive attitudes towards Spanish, followed by Catalan and the least positive attitudes towards English. The main reasons are to be found in political, 
social and psychological issues. More specifically, we have found how the sociolinguistic status of a language may heavily influence attitudes to languages.

(Portolés 2015, 172)

Ahí vemos de qué modo lo que de manera objetiva constituye una realidad social (la presencia de determinadas lenguas en un entorno comunicativo) puede ser vivido diversamente de manera subjetiva por cada uno de los miembros de esa comunidad. Esta otra dimensión es la que ha permitido a Block (2006) poner el multilingüismo en relación con la identidad frente a la globalización y los fenómenos migratorios. Y a Schwieter (2017) estudiarlo más bien desde una perspectiva cognitiva y perceptiva, que es la que está más en contacto con la definición de multicompetencia lingüística con la que comenzábamos. Finalmente, donde se ofrece una exhaustiva visión de la complejidad inherente a la competencia multilingüe es en The Cambridge Handbook of Linguistic Multi-Competence (Cook y Wei 2016). Sin embargo, es cierto que no ha sido puesta en relación directa con el humor, que es el tema que aquí nos atañe, aunque sí se ha analizado con respecto a la emoción. En ese sentido, son clarificadoras las conclusiones a las que llega Dewaele $(2016,474-5)$, que veremos también en cierto modo reflejadas en las narraciones que hemos analizado en este artículo:

The addition of a language (or a culture) to an individual's repertoire has profound repercussions. On the whole system, including the individual's emotional geography. The acquisition of new emotion concepts, and of new sociopragmatic and sociocultural information governing their use, affects the L1 emotion concepts and the way they are verbalised. [... The multilinguals] typically rate their $\mathrm{L}_{1}$ as being more emotional than their LX. However, there are clear instances of blending of L1 and LX values and practices. While they may be able to keep their languages apart in interactions, more permeability develops between the two languages at a conceptual level. LX affective socialisation results in a unique multicompetent behavior both in the L1 and LX. Swearing in the LX illustrates the newfound freedom to express oneself without violating L1 norms. The new language and culture offer LX users new potential emotional selves which they can deploy according to their needs.

(Dewaele 2016, 474-5)

En los estudios generales sobre la psicología del humor, paralelamente, se ha correlacionado este con las emociones (Martin 2007, 30-33), pero no en particular con el modo en que el humor pueda verse afectado por la multicompetencia lingüística de los hablantes. Para poner en relación este aspecto con los estudios sobre el humor desde una perspectiva lingüística, hay que decir que debemos a Timofeeva-Timofeev (2014) uno de los primeros acercamientos al humor verbal infantil en español, en particular en la etapa de educación primaria. Posteriormente, Timofeeva-Timofeev (2016) ha estudiado el uso de la fraseología por

\section{2nd proofs}


parte de este colectivo con fines humorísticos. En uno de sus últimos trabajos (Timofeeva-Timofeev 2017) ha delimitado teóricamente el campo de estudio de la metapragmática del humor infantil, para analizar las marcas e indicadores lingüísticos del humor, previamente identificados por Ruiz-Gurillo (2012), que aparecen en las narraciones de niños y niñas de entre 8 y 12 años del corpus CHILDHUM, desde diversas perspectivas. Entre ellas, la identidad de género a través del humor (Galindo Merino 2017); las figuras retóricas como estrategia para construir humor (Marimón 2017); la expresión de la subjetividad mediante elementos valorativos, atenuadores e intensificadores (Martínez Egido 2017); los idiomas inventados que aparecen en estas narraciones (Ruiz-Gurillo 2017); y el humor infantil a través de la formación de palabras (Santamaría 2017). Los fundamentos teóricos acerca del desarrollo de la habilidad metapragmática del humor lingüístico establecen que esta capacidad se va adquiriendo paulatina y acumulativamente, atravesando desde la infancia una serie de etapas que fueron ya descritas por Gombert (1992). Así, en la interpretación de este y siguiendo a Timofeeva-Timofeev (2017), si hasta los 2 años se adquieren las habilidades lingüísticas básicas, entre los 2 y los 7 años el niño o la niña empiezan a ser conscientes de la conexión entre lo lingüístico y lo contextual, aunque sin distinguir completamente todavía ambas dimensiones. Finalmente:

A partir de los 7 años aproximadamente y hasta los 12 los infantes se sitúan en el tercer estadio de desarrollo, donde aprenden a discernir entre el mensaje lingüístico y el contexto. Ello les capacita para comenzar a controlar la conexión entre ambas informaciones en el uso y a describir explícitamente cómo se lleva a cabo dicho control. Se adquiere, por tanto, la conciencia metapragmática, tanto desde la perspectiva interpretativa como la productiva. (Timofeeva-Timofeev 2017, 8)

Esta es la fase en la que se encuentran los informantes del presente estudio. Así pues, tras este breve repaso al estado de la cuestión, y como contribución a esta línea de trabajo, en este artículo nos planteamos las siguientes preguntas de investigación:

1. ¿Se producen cambios de código que muestren multicompetencia lingüística en las narraciones humorísticas infantiles de nuestro corpus? En caso afirmativo, ¿con qué frecuencia aparecen? ¿Cuáles son las lenguas intercambiadas?

2. ¿Puede interpretarse la inclusión de palabras o expresiones en otras lenguas distintas a aquella en la que están redactadas las narraciones, es decir, el español, como el resultado de una voluntad manifiestamente humorística? Dicho de otro modo, ¿puede deberse el cambio de código al deseo de generar humor, consciente o inconscientemente? En caso afirmativo, ¿de qué tipo de humor se trata? En caso negativo, ¿a qué otra dimensión obedece? 
3. ¿Se hace referencia en tales narraciones a la diversidad lingüística y el multilingüismo? En caso afirmativo, ¿de qué modo? ¿Qué actitudes se reflejan?

Hemos intentado responder a estas cuestiones a la luz del análisis detenido del corpus que veremos en el tercer apartado, pero antes mostramos a continuación la metodología empleada para el desarrollo del estudio.

\section{Metodología}

Hemos trabajado a partir del corpus CHILDHUM, resultado del proyecto de investigación dirigido por Larissa Timofeeva-Timofeev, Metapragmática del humor infantil: adquisición, perspectiva de género y aplicaciones (GRE14-19, Universidad de Alicante). Este corpus consta de 448 narraciones de tema humorístico escritas por niños y niñas de 8,10 y 12 años procedentes de cinco colegios de la provincia de Alicante. El corpus CHILDHUM se encuentra actualmente en expansión al amparo del nuevo proyecto La formación de la conciencia figurativa en la etapa de Educación Primaria: el humor y la fraseología (FFI2016-76047-P, AEI/FEDER, UE). A través de este último no solo se pretende ampliar las muestras, sino también las perspectivas de análisis desde las que enfocarlas. Puede hallarse en Timofeeva-Timofeev (2017, 5-19) una descripción minuciosa del procedimiento de recogida y etiquetado del corpus, con toda la información acerca del tipo de centro (público o privado), la zona de predominio lingüístico (valenciano o castellano), el programa lingüístico seguido en la escuela (de inmersión en valenciano o no), las variables edad y género, así como la justificación tanto de la modalidad escrita para la recogida de datos como del tipo de redacción que se les pidió a los participantes.

A partir de este corpus global, hemos seleccionado para nuestro estudio un total de 184 narraciones (un $41 \%$ del total de CHILDHUM) que son las que mostraban ejemplos, bien de cambios de código, bien de referencias al multilingüismo. En concreto, este corpus de 184 narraciones se reparte según las franjas de edad del siguiente modo: 41 escritas por niños y niñas de 8 años, 80 de 10 años y 63 de 12 años, tal como se refleja en el Gráfico 1 a continuación: ${ }^{4}$

En los dos primeros casos (8 y 10 años), la narración escrita consistía en describir cómo sería un intercambio escolar de dos semanas en Marte; en las pautas que recibían los niños se les indicaba que contaran cómo sería su día a día, la relación con los compañeros, las asignaturas, el profesorado, la manera de comu-

4. Con respecto al conjunto del corpus CHILDHUM, estas cifras representan el $30 \%$ de las narraciones de 8 años, el $60 \%$ de las de 10 años y el $40 \%$ de las de 12 años.

\section{2nd proofs}




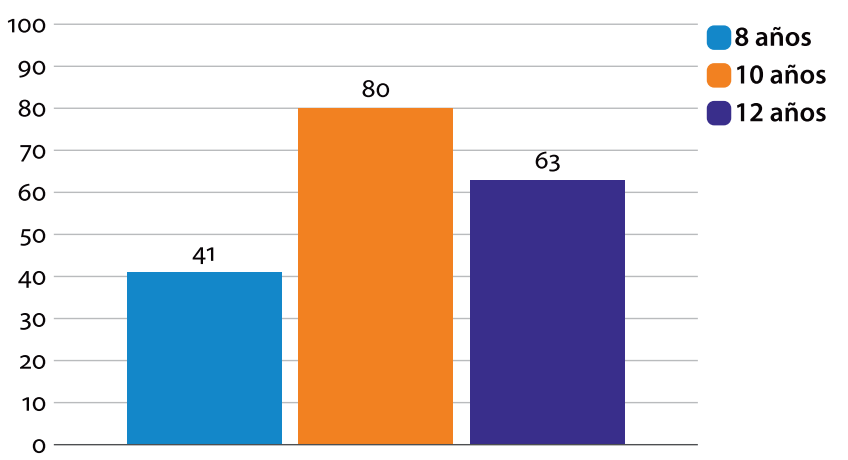

Gráfico 1. Narraciones del corpus según franja de edad

nicarse, etc. Por el contrario, los participantes de 12 años tenían que describir cómo sería su vida después de despertarse habiéndose convertido en un insecto. En ambos casos se les pedía que intentaran que su historia fuera divertida, por lo que hay en ellos una intención humorística de partida.

Los aspectos relacionados con la multicompetencia lingüística en las narraciones que hemos analizado son de dos tipos:

a. Cambios de código, con la aparición de términos o expresiones en otras lenguas.

b. Referencias a otros idiomas aparte del castellano (que es la lengua en la que están escritos los textos) y comentarios sobre ellos.

A continuación, presentamos la discusión de los datos obtenidos, tanto de un modo cuantitativo como cualitativo, mostrando abundantes ejemplos ilustrativos.

\section{Análisis y discusión de resultados}

Como se indicaba previamente, para llevar a cabo este estudio hemos partido de las narraciones escritas de 184 niños y niñas de entre 8 y 12 años que permitirían ver la evolución del desarrollo metapragmático del humor en relación con su competencia plurilingüe. Tras la lectura detenida de todas estas redacciones, hemos encontrado ejemplos de multicompetencia lingüística de diversa índole y en distintas proporciones según las edades. Por ello hemos considerado oportuno irlos mostrando y comentando según ese criterio cronológico, porque tal vez así se intuya cómo van evolucionando las muestras. De todos modos, entre los aspectos relacionados con la multicompetencia lingüística que hemos encontrado y que decíamos que eran básicamente de dos tipos, sí hemos visto que se repiten algunos patrones:

\section{2nd proofs}


1. En los casos de cambios de código, los términos o expresiones en otras lenguas obedecen a (a) extranjerismos (fundamentalmente anglicismos, aunque no solo); (b) antropónimos en otras lenguas para nombrar a los personajes de la historia contada; y (c) valencianismos, como resultado del bilingüismo español/valenciano.

2. Por lo que respecta a las referencias a otros idiomas, hemos encontrado, por un lado, menciones a diversas lenguas como materias de asignaturas (solo en las narraciones de 8 y 10 años, no en las de 12, probablemente a causa del tema de la redacción). Y por otro, comentarios muy sustanciosos sobre la comunicación entre hablantes de diversos idiomas, o sobre la facilidad o dificultad de aprendizaje de segundas lenguas, que reflejan la actitud ante la diversidad lingüística.

Seguiremos este orden en el comentario de los ejemplos, mostrando la evolución por grupos de edad.

\subsection{Multicompetencia lingüística en el grupo de 8 años}

Comenzando con este primer grupo, de las 41 narraciones analizadas, los ejemplos obtenidos en cada una de las categorías descritas se reparten tal y como se especifica en el Gráfico 2 ( 57 \% Comunicación, 16 \% Valencianismos, 12 \% Lenguas en asignaturas, $10 \%$ Antropónimos y $5 \%$ Extranjerismos):

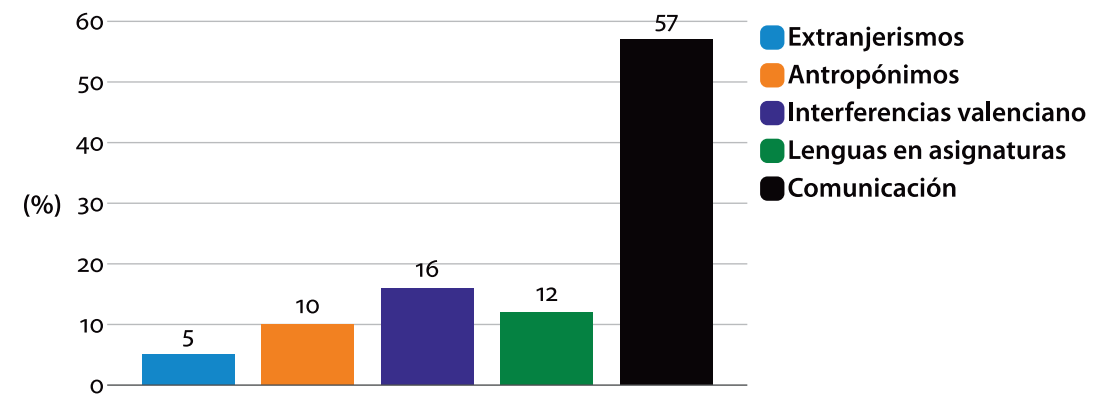

Gráfico 2. Grupo de 8 años

En este grupo los ejemplos de extranjerismos $(1,2,3,4)$ son escasos, probablemente porque el conocimiento de otras lenguas aún no está lo suficientemente asentado; en algunos casos hacen referencia a términos deportivos, y en otros incluyen, o bien algún ejemplo de sufijación pretendidamente humorística (culoman, marcienglis), o bien términos (friqui, basquet, badminton) que en realidad ya han sido aceptados por la RAE: 
(1) 8, 14-15 AO37: Jugaban a los bolos con lechugas y cocos. Cuando jugaban al futbol usaban rollos de papel evez de balones de futbol. Eran friquis. ${ }^{5}$

(2) 8, 14-15 BO6: Jugamos a la pelota, a badminton y a basquet [...] Alli hay un profesor que se llama culoman.

(3) 8, 14-15 BO7: en gimnastica carreras de minicars de fuego [...] Tirando pizzas fanta y planetas por todo Marte [...] Pista de carts con carts propios y pistoletas de gomas [...]

(4) 8, 14-15 SO8: "Las asignaturas marcilegua, marcimates, marcienglis, marcispikin"

Un aspecto curioso, que como veremos se repite en el resto de grupos, es el hecho de que utilicen otras lenguas distintas al español para denominar a los personajes de su historia (recordemos que se les pedía que narraran cómo sería una semana de intercambio escolar en Marte). Entre los antropónimos en otras lenguas predominan los ingleses, probablemente por influencia de películas o dibujos animados anglosajones, pero también aparecen algunos inventados. Podemos verlo en los siguientes ejemplos (5) a (10):

(5) 8, 14-15 AA27: Y por último el recreo: el balón es la tierra, la comba son los rayos del sol y el tobogán eran los anillos de Saturno. Y los marcianos uno se llamaba Rudi tenía la cabeza roja y Tubi tenía los pies amarillos

(6) 8, 14-15 MO14: Un niño que se llamaba Eslot era mi amigo

(7) 8, 14-15 SA3: ¡Rin! ¡Rin! me mandaron un mensaje, mire al telefono y... ¡Salio mi mejor amigo, Llefri! Fui corriendo a Marte y me dijeron que estaban en apuros y no dije nada. Llefri estaba triste y yo tabien.

(8) 8, 14-15 SO10: "Mi nombre era combo y como apellido Tam y Comba"

(9) 8, 14-15 BA2: La profesora se llama Margaret

(10) 8, 14-15 BO14: Mis profesores son Luke y Elisabet

Respecto a las interferencias lingüísticas del valenciano (variedad dialectal del catalán que toma esta denominación en la Comunidad Valenciana de la que se extrajo el corpus), no es de extrañar que, en este grupo como en los restantes, aparezcan con mucha mayor frecuencia en las narraciones obtenidas de centros con programas multilingües o de línea en inmersión en valenciano. Aquí, el cambio de código entre las dos lenguas que se adquieren de manera bilingüe y cuya lectoescritura está en proceso de afianzamiento es un fenómeno normal y ampliamente

5. Todos los ejemplos han sido transcritos literalmente.

\section{2nd proofs}


estudiado. Por un lado, vemos sobre todo ejemplos (11) a (15) de interferencias involuntarias ( $i$ por $y, x$ por $c h$, traer por sacar, charrar por hablar...):

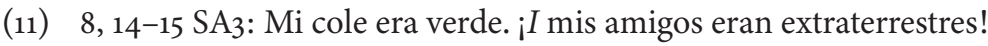

(12) 8, 14-15 $\mathrm{BO}_{4}$ : He visto 4 compañeros y los he conocido: uno se llama Pepito una Pepita, uno Alberto $i$ la otra Inés $i$ habian 6 profesores y se llamaban... [...] A la hora del patio almorcé un bocata de salmon $i$ jugue a la comba $y$ al futbol" [...] Al dia siguiente estudie $i$ hice el control de matematicas $i$ trae un 8'6 (trae por saqué) i despues jugue a la comba $i$ al futbol $i$ despues dine (dine por comí) $i$ me fui al bosque de marte a jugar con mis compañeros

(13) $\quad 8,14-15$ BA2: al que se porta mal le regala xuxes [...] Viven en xampinones gigantes

(14) 8, 14-15 MO14: cuando veia el chocolate decía xocolati o me da una xocolatina o te mato gallina

(15) 8, 14-15 AA17: Mis compañeros charran mucho (charrar por hablar)

Paralelamente, hemos encontrado algunos términos coloquiales en valenciano con una intención más claramente humorística, como los que vemos en el ejemplo 16:

(16) 8, 14-15 MO14: El niño se cabrea y le canta una nana a su maestra, la maestra se cabrea más y la maestra coge al pobre ñaco de las piernas y hace como lo baquero. ¡Hijo! Y tira al niño por la ventana. Un ñaco es más pesao que la leche [...] Un nino se tiro una chufa [...] Para despegar me tire un petorro y para aterrizar a la tierra me tire una bufa.

Con respecto a las referencias textuales a otras lenguas, en 7 de las 41 narraciones $(17 \%)$ hemos encontrado alusiones a tres idiomas básicamente (inglés, valenciano, lengua española) entre las asignaturas mencionadas dentro de su relato (recordemos que contaban un intercambio 'escolar' en Marte), lo cual sin duda reproduce su realidad más inmediata. En algún caso, con curiosas variaciones, como se advierte en el Ejemplo 17:

(17) 8, 14-15 AA19: Las asignaturas alli era asi lengua lengüeta mates matis musica musiley ginasia giniasita cono conito valenciano valen ingles inglis pitinglis plastica plastiquis

Más interés, en cambio, ofrecen los comentarios acerca del idioma extranjero (real o inventado) como medio de comunicación en el contexto del relato, que aparecen hasta en un $80 \%$ de los textos de este grupo. Hay que tener en cuenta que en las instrucciones se especificaba "Cuéntanos cómo te comunicas con tus amigos marcianos, cómo es el idioma en que hablas con ellos, etc.” A esta edad

\section{2nd proofs}


hay ya una conciencia clara de que en lugares distintos se hablan diferentes lenguas, por eso hay muchas referencias a idiomas inventados (para cuya descripción y análisis nos remitimos a Ruiz-Gurillo 2017) y, en menor medida, a otros idiomas: a veces lenguas conocidas, a veces un idioma supuestamente propio de Marte. Las referencias a la lengua en la que hablan los extraterrestres se relacionan con un elemento de extrañamiento que esconde en muchas ocasiones un componente humorístico. Todo ello remite a su percepción del multilingüismo, a la conciencia de la existencia de otras lenguas distintas a la propia, que además pueden ser aprendidas. En ese sentido, se advierte una actitud positiva hacia su aprendizaje, como puede verse en los Ejemplos (18) a (25): 6

(18) 8, 14-15 AO41: El idioma era asi $\wp \mathfrak{J}$ [signos indescifrables]. Esto mismo apa-

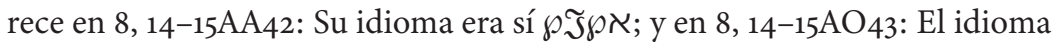
era asi $\wp \mathfrak{J} \wp$.

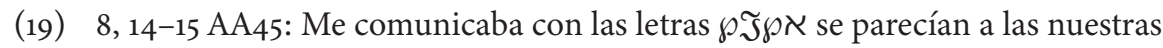
[cuatro símbolos inventados, inidentificables). Hola se decía

(20) 8, 14-15 AO46: א א א [y por debajo, como traduciendo] como te llamas asi que aprendi a abar a su ioma.

(21) 8, 14-15 AO40: Érase una familia que vivía en Londres y decidieron ir al centro espacial y cogieron un cohete para ir a Marte en Marte fueron a un colegio y aprendió muchas cosas su idioma era 汉字 汉字汉字 [indescifrable, parecido a chino...] la comida era siempre de primero 汉字 de segundo 汉字 y de postre 汉字 y cuando pasaron las dos semanas se volvieron a Londres!

(22) 8, 14-15 AO4: Del idioma que hablan en marciano pero estudié mucho su idioma así que ya se su idioma

(23) 8, 14-15 AA18: ¿Podemos ser amigos? Y les decian bla blabla blabla bla porque abalaban en no se eran extraterrestres

(24) 8, 14-15 AA19: Hola como te llamas ella no me contesto y me acorde de que ella no ablaba mi idoma pero enseguida aprendi el suyo se comunicaban asi babada babada era un dioma muy raro pero enseguida lo aprendi

6. Un único caso de los 33 encontrados refleja una actitud negativa hacia la diversidad lingüística: 8, 14-15 AA22: mis compañeros ablan en el idioma ta ta ta ablan asi mu raro [...] avia un teatro que ivan hacer un teatro llamado tatararara yo no lo entendio ni a mis compañeros estaba deseando bolber al oto mundo [...] al dia siguiente bino otra chica que era de Elda ella tampoco entendio nada eramos las dos las mejores amigas [...] era un rollo estar en Marte estaba deseando ver a mi familia menos mal que tenia a mi amiga jugabamos y a ella si que la entendia porque ella hablaba en español y van hacer un teatro.

\section{2nd proofs}


(25) 8, 14-15 BA2: Me enseñé su idioma es gracioso

En otros casos, las lenguas de comunicación que se mencionan son el inglés, inglés con español, inglés con italiano, chino, japonés, español y valenciano, portugués, alemán... Pero en todos los casos, también cuando se menciona la 'lengua marciana', lo más relevante es que la diferencia lingüística no provoca incomprensión, como puede verse también en los Ejemplos (26) y (27):

(26) 8, 14-15 BO9: Estoy desde hace mucho tiempo en una escuela de marte y hablo un idioma japones que entienden.

(27) 8, 14-15 IA20: Y los marcianos hablaban asi bling blang blung bleng los marcianos nos entendian y leian el celebro.

Es como si se partiera de la evidencia de la comunicación real independientemente de la lengua en la que hablemos, de manera que esta es vista como un instrumento que facilita (y no que dificulta) nuestra intercomprensión.

Pasamos a comentar ahora los resultados obtenidos en el grupo de 10 años.

\subsection{Multicompetencia lingüística en el grupo de 10 años}

En el Gráfico 3 podemos ver reflejados los porcentajes en que se presentan en este grupo de edad los fenómenos que estamos estudiando (64 \% Comunicación, 15 \% Extranjerismos, 8 \% tanto Lenguas en asignaturas como Valencianismos y $6 \%$ Antropónimos):

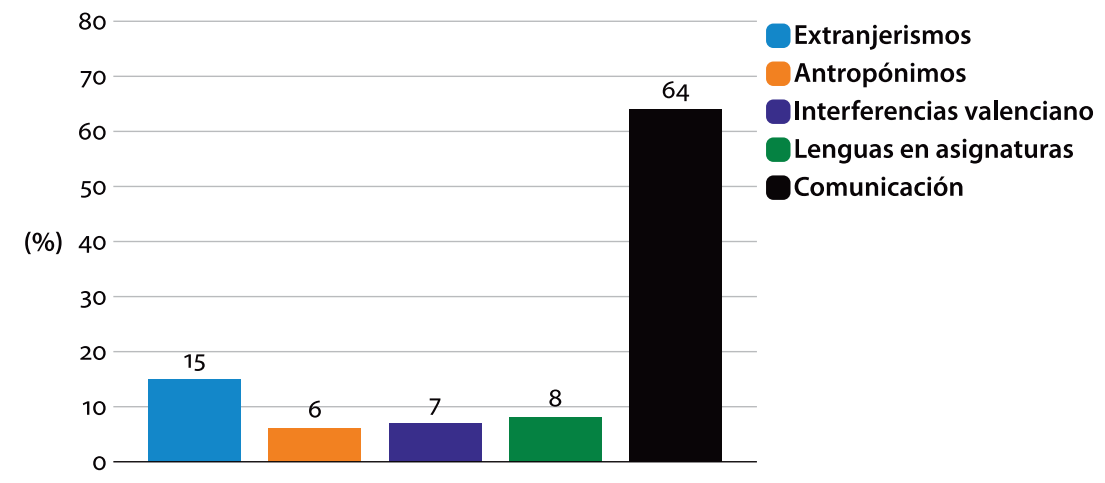

Gráfico 3. Grupo de 10 años

Según el orden seguido hasta ahora, cabe decir que el porcentaje de extranjerismos aumenta (un $8 \%$, frente al $3 \%$ anterior), tendencia que se confirmará en el siguiente grupo, como veremos, lo que probablemente obedece a una pro- 
gresiva ampliación de la competencia plurilingüe de los informantes. Veamos los Ejemplos (28) a (37), muchos de los cuales hacen referencia a deportes, comidas o marcas y tienen una función meramente descriptiva:

(28) 10, 12-13 SA9: Nos habian dejado en un hotel cinco meteoritos jera una pasada! Habia llacusi, zona de juegos, bufet de chocolate...

(29) 10, 12-13 $\mathrm{SA}_{3}$ : A mi me gusta peperoni

(30) 10, 12-13 SA6: Cuando llegue al espais class [...] Yo pedi: nueve nuguet del macdonal y una coca-cola, Paula se pidio piza de cuatro quesos

(31) 10, 12-13 SO12: Tenia una "jetpack" incorporada en el traje

(32) 10, 12-13 SO13: Todos mis amigos tiene Clash of Clash

(33) 10, 12-13 AO31: Me comunico con mis amigos marcianitos con un movil muy raro que se llama tipali es tactil y no tiene tuenti tiene tuemul.

(34) 10, 12-13 SA4: Llevaba unas gafas como las de "Harry Potter" y unos braquets rojos muy chulos

(35) 10, 12-13 IO43: jugamos a fulonmano, voleyhandbol

(36) 10, 12-13 SO18: Estuvimos jugando a la Play Station

(37) 10, 12-13 IO4: ¡FIN! THE END / 10, 12-13 IO3: FIN THE END

De nuevo encontramos también casos de antropónimos en otros idiomas, en menor proporción que en el grupo de menor edad ( $\tan$ solo en un $8 \%$ de las narraciones), si bien con una intención humorística, como puede observarse en los Ejemplos (38) a (41):

(38) 10, 12-13 SA2: Vamos a cantar la Milésima sinfonía [...] Hecha por Lucky One Desafinohuen. [...] Una niña parecida a una humana que se llama "Ricky galaxian".

(39) 10, 12-13 SA11: La directora es perfida y malvada. - Como se llama, "Mister Chiflada".

(40) 10, 12-13 SA23: Mi profesor se llamaba Potato.

(41) 10, 12-13 SA27: Hoy daremos los planetas dijo el señor Brain (el profesor)

En cuanto a los ejemplos de interferencia con el valenciano (42 y 43), se limitan también a los centros bilingües y no advertimos en ellos ninguna intencionalidad humorística. Junto a los casos ya vistos de $i$ por $y$, aparecen ahora con frecuencia -ava por -aba en la terminación verbal del imperfecto, ss por $s$ y $n y$ por $\tilde{n}$, entre otros:

\section{2nd proofs}


(42) 10, 12-13 BA11: habia una maestra que venia de espanya y sabia hablar espanyol. [...] la senyorita Paqui [...] un extraterrestre muy guapo, carinyoso '... esto no me lo ensenyo yo ni en tres anyos [...] professor [...] entropecé en una piedra [...] le pege un beso a mi novio extraterrestre

(43) 10, 12-13 BO9: Me despido de todos $i$ espero en la nave para subir. En la tierra lo cuento todo $i$ nadie me entiende $i$...

En uno de los casos (Ejemplo 44), el relato comienza en castellano (con abundantes interferencias) y termina en valenciano, de manera natural, inconsciente, un fenómeno usual en contextos bilingües, especialmente en la lengua oral:

(44) 10, 12-13 BO1: cuando llegamos ha marte no saviamos hablar marciano. Peró al largo del tiempo nos ensenyamos $i$ empecamos ha hablar despues era todo tan precios. [...] No dabamos clase en la classe si no en el patio. I los ordenadores eran muy estranyos eran redondos $i$ muy pequenyos [...] I no nos pegaban corchotadas [...] cuando nos caiamos nos pegavamos con una piedra mos feem mal en el cap perque no duiem casco. Pero tampoc duiem pantalons, ni camises, ni calzetins, tampoc camisa llarga [...] hacia mucha calor.

Por lo que respecta a las referencias a otros idiomas como contenido de asignaturas, en este grupo de 10 años no aparecen más ejemplos que en el anterior (solo un $11 \%$ ), pero a cambio las lenguas mencionadas son más variadas: valenciano, castellano, chino, japonés, inglés, francés, catalán, y también idiomas del mundo terrestre y lengua marciana.

Sin embargo, lo que sí es realmente muy interesante son los numerosísimos ejemplos (hasta en un $91 \%$ de las narraciones) de comentarios acerca de la diversidad lingüística, las lenguas de comunicación (entre las que aparece ahora la lengua de signos, que en ocasiones es motivo de risa), la facilidad o dificultad en el aprendizaje de las diferentes lenguas, la manera de comunicarse y los sentimientos de diversión o frustración ante la posibilidad o no de entenderse (Ejemplos 45 a 76). Veamos algunos de ellos, comenzando por aquellos más escasos (45 a 47) en los que una lengua distinta es algo confuso y negativo, que provoca un choque lingüístico y que se aprecia como algo difícil:

(45) 10, 12-13 IO37: Cuando entre no entendia nada hablaban marciano un idioma superdificil de aprender.

(46) 10, 12-13 IO28: Todo es muy aburrido no puedo hablar con nadie mis compañeros son muy feos no entiendo a los profesores no haprendo nada no puedo respirar no manda dederes todos hablan en una lengua muy rara.

(47) 10, 12-13 IA26: Inglés será inglés pero con otro idioma ¡Que lio por favor! [...] y el idioma es lo que mas me preocupa [...] Ya me apallaré con el idioma madre-

\section{2nd proofs}


mia ¿Cómo nos comunicaremos? Y si tengo que decirle algo urgente a la profesora/or y a mis amigos/as como será será escrito, escrito no no entienden la letra del planeta tierra [...] Como el idioma son diferentes tendre que enseyarlos yo a ellos o ellos a mí, a mi me parece más fácil que yo les enseye a ellos y asi ellos aprenden mi idioma y el suyo.

En otros casos (48 a 52), esa incomprensión y confusión se salva porque 'ellos' saben 'nuestro' idioma:

(48) 10, 12-13 SA28: Menos mal que los profesores supuestamente sabian un poco nuestro idioma [...] los marcianos eran simpáticos sabian tambien un poco de español.

(49) 10, 12-13 BA13: Cuando llegamos a Mart nos hablavan muy raro nos decian cosas estrañas. No entendemos ni papa de lo que decian los marcianos [...] aveces tambien intentavan hablar nuestro idioma.

(50) 10, 12-13 IO7: En este colegio hablan con un lenguaje muy dificil pero tambien hay algunos que hablan español.

(51) 10, 12-13 BO25: Jo les pregunte que si me entendian i en mi idioma me respondieron que si osea que entendian mi idioma.

(52) 10, 12-13 IO32: ¡Guau! Entiende nuestro idioma. Según mi diccionario todos los marcianos verdes entienden nuestro idioma.

El hecho de hablar por gestos, a los que se alude en diferentes narraciones ( 53 a 56), es visto como una situación divertida, que genera humor, de manera que la incomunicación no es conflictiva, sino que, al contrario, provoca risa:

(53) 10, 12-13 IA39: Yo me comunicaba con los compañeros con señas con las manos ellos no me entendían muy bien. Los compañeros se reían conmigo porque no me entendían nada con las señas y yo me reía porque su risa era muy graciosa. Yo hablaba con ellos en español y ellos se reían porque no entendían nada de nada, su idioma era muy raro y extraño y también gracioso.

(54) 10, 12-13 IO22: Con mis amigos marcianos me comunico con jestos es muy divertido.

(55) 10, 12-13 $\mathrm{AO}_{39}$ : Me comunico con mis amigos marcianos haciendo jestos o juntando las frentes, hablan en el idioma la la lela lele leli i ademas lo dicen con gracia su idioma.

(56) 10, 12-13 SO22: aunque no se me el idioma marciano me costaria mas pero igualmente me podria comunicar con gestos. ¡Seguro que seria todo super divertido! 
Otro aspecto novedoso con respecto al grupo anterior son las referencias (57 a 61) a tecnologías que permiten la comunicación:

(57) 10, 12-13 MO2: Me comunico con los marcianos con un comunicador Xfair7.

(58) 10, 12-13 $\mathrm{AO}_{31}$ : Me comunico con mis amigos marcianitos con un movil muy raro que se llama tipali es tactil.

(59) 10, 12-13 IO18: Yo me comunico por un traductor alienígena y también le hago señales y le hablo un poco al tun tun tun.

(6o) 10, 12-13 AO21: Nos comunicamos a traves de unas antenas que todos tenemos para hablar en cualquier idioma, normalmente hablamos en idioma universal.

(61) 10, 12-13 AA28: Como no entendiamos nada nos dieron un aparato que nos poniamos en el oido para entender a los marcianitos y otro en el cuello para poder comunicarnos.

En la mayoría de las ocasiones (62 a 76), lo cual es significativo, el nuevo medio de comunicación es visto como algo curioso, divertido, a lo que rápidamente te acostumbras, que no ofrece obstáculo, que no dificulta en el fondo la comunicación:

(62) 10, 12-13 SA8: Julia era otra compañera mía era muy simpatica y lo malo era que ¡no! ¡entendía! ¡su! ¡idioma! (a continuación se dan ejemplos con símbolos inventados) [...] Fui a la cantina del cole y vi a una china que decía: solaholachanis sileno quesohe chamarrachi cometulo: Que significa hola jovencita quieres un carameliko de fresa, y le dije que si sabeis me hablaba en chino y yo se chino

(63) 10, 12-13 IO12: me comunico por el telefono mobil y hablamos idioma japones $y$ chino [...] tambien hablamos idioma marciano.

(64) 10, 12-13 BO7: Jo no los entendia [...] para entenderlos me apunte a un cursillo. El primer dia de colegio me fue fatal. El segundo dia me fue mejor ja hablaba mejor i lla los entendia. Resultava que escribian con signos i como no lo sabia el primer dia me fue mal. Ahora no me parecia su idioma raro i el idioma no me causo mas problemas.

En ocasiones, aunque no se haga alusión directa a la lengua en que se comunican, sí se describen conversaciones enteras entre un idioma inventado y el español como hace el informante (10,12-13 IA15), con lo cual se da a entender indirectamente que no existen problemas de comunicación. En general, en la mayoría de las narraciones se da por sentado que se aprende con facilidad la nueva lengua o que al menos es posible hacerlo, que resulta fácil la comunicación y, sobre todo, divertida:

\section{2nd proofs}


(65) 10, 12-13 $\mathrm{IO}_{3}$ : Nos comunicamos tocando musica porque cada parte de su cuerpo suena como un instrumento [...] Tambien nos comunicamos haciendo gestos [...] Muchas veces hablamos marciano y otras como yo español. Hay algun niño de marte que save todos los idiomas de la tierra y marte. Hay un niño como yo que es el unico que me entiende pero la verdad es que da igual, este cole es superdivertido.

(66) 10, 12-13 SA16: ¿Y te preguntas como nos comunicamos? Pués muy facil yo le enseñaba mi idioma y ella el suyo.

(67) 10, 12-13 MO3: Mis amigos marcianos hablaban con gestos, moviamos las manos hacia arriba y hacia abajo y señalaba lo que queria lo más guai era que hablábamos con la mente. ¡Y era muy divertido!

(68) 10, 12-13 SA11: Pero como voy a avanzar en este colegio si ni siquiera se la lengua "Moroque" - Te acostumbrarás.

(69) 10, 12-13 SA1: Normalmente los marcianos hablan los idiomas raros al principio del todo no les entendí es chulo su idioma al principio del todo era dificil entenderlo. Pasado el tiempo fui conociendo amigos. [...] en las dos semanas se pudo aprender el idioma.

(70) 10, 12-13 IA5: Lo que mas me ha asombrado era el idio en el que hablaban ¡me ha costado un monton aprenderlo!, pero pol lo menos soy bilingüe porque hablo español y marciano.

(71) 10, 12-13 $\mathrm{AO}_{3} 6$ : Nos comunicamos chocando la palma de la mano y el idioma es muy raro pero yo puedo hablar con ellos es muy fácil.

(72) 10, 12-13 MO8: Aunque al principio no sabia ablar su idioma asi que no podia con ellos. Asi que me comunicaba con gestos. Al final, al final, hal final ablaba con ellos con su lengua oficial.

(73) 10, 12-13 $\mathrm{SO}_{33}$ : Tambien tendre profesores seguro que me alludaran a hablar en su idioma [...] yo me comunicaria con metiorisitas o escribira en un folio una carta. Hablo con ellos con gestos si me entiende pero si no me entienden buscaria palabras en su diccionario y se lo escribo. Hablar con ellos seria divertidisimo porque conoceria su lengua.

(74) 10, 12-13 SO35: Mis amigos marcianos y mis amigos incluido yo nos comunicamos asi llo tener 10 años o tu tener 9 años o tambien nos comunicamos de otras formas [...] mola su idioma un montonazo.

(75) 10, 12-13 AA27: hablo o me comunico haciendo gestos y no se como me sale pero los profesores me entienden y mola mucho. 
(76) 10, 12-13 BA1o: Yo me quede flipada con el idioma [...] es marciano de tenia al lado era simpatiquisimo con el aprendi a hablar marciano en seguida

En definitiva, como se ha mostrado, en la mayoría de las narraciones, la diversidad lingüística es vista como algo positivo, que genera diversión, a pesar de la extrañeza o dificultad de comunicación inicial, y que puede por tanto provocar humor. Ello muestra, en suma, una buena predisposición hacia el multilingüismo, una cierta curiosidad hacia otras lenguas y una buena disposición a aprenderlas. Veamos por último los resultados del tercer grupo de edad.

\subsection{Multicompetencia lingüística en el grupo de 12 años}

En las narraciones humorísticas del grupo de estudiantes de 12 años, los fenómenos que estamos comentando aparecen distribuidos en el Gráfico 4 del siguiente modo (Extranjerismos 38 \%, Antropónimos 29 \%, Comunicación 19 \%, Valencianismos $14 \%)$ :

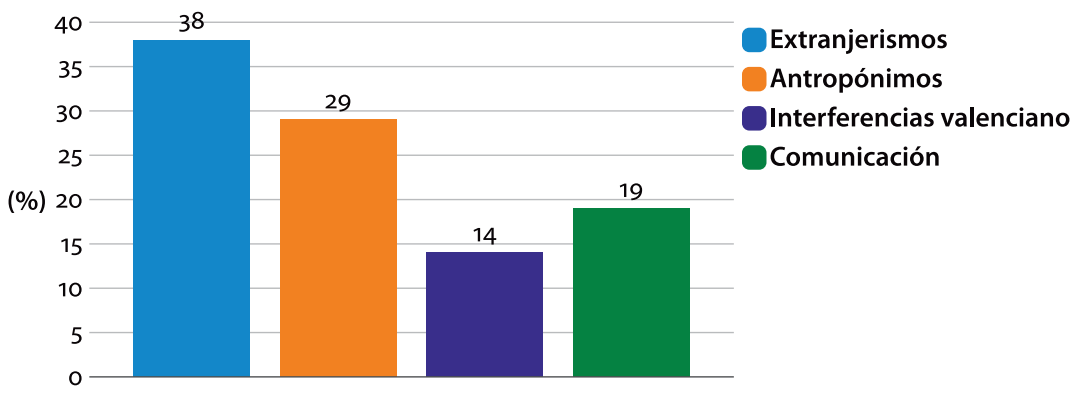

Gráfico 4. Grupo de 12 años

Destacamos, en primer lugar, que los ejemplos de cambios de código a través de extranjerismos aumentan significativamente respecto a los anteriores grupos (aparecen hasta en un $38 \%$ de las narraciones), probablemente como resultado de una mayor competencia en lengua extranjera. Por un lado, aparecen frecuentemente en los títulos y las despedidas, y por otro, referidos básicamente a fiestas extranjeras, marcas, películas, personajes infantiles, juegos, deportes, comidas, lugares o canciones, ejemplos todos ellos que muestran su competencia intercultural plurilingüe, aunque no advertimos intencionalidad humorística alguna, como puede verse en los Ejemplos (77) a (89):

(77) 12, 14-15 $\mathrm{IO}_{4}$ : ...me insultaron del traje que había llevado, normal que serrían era Hallowenn [...] pusieron la canción del Gam Gam Style [...] mi madre llamo a Carglas

\section{2nd proofs}


(78) 12, 14-15 Bo7: KING BEE [título]

(79) 12, 14-15 IO18: Nada más levantarme me puse a bailar, tenía mucho, pero que mucho muchísimo FLOW

(8o) 12, 14-15 BA3: Esta mañana he desayunado rechbul y ya sabes que te da alas. [...] THE END [Al final]

(81) 12, 14-15 SA35: Una especie de Indian Jones la atrapó por detrás [...] te metiste dentro de un patín de Barbie

(82) 12, 14-15 IO17: Me fui a mi casa a hechar unas partidillas de Call of Duty Advanced Warfare

(83) 12, 14-15 SA35: "Gimnasio Polen Sport"

(84) 12, 14-15 BA6: Donde vivian las moscas era MINICITY

(85) 12, 14-15 MA8: Entonces nos sirvió su risotto

(86) 12, 14-15 AO11: ACERTIJO 1 (¡los acertijos los pongo in spanish!) [...] Le mande un Wats App a mi madre

(87) 12, 14-15 SO29: El mago vive en "Wizard street" (los 4 personajes de la historia tienen nombre inglés)

(88) 12, 14-15 MA9: ¿Quién compuso la canción La dona e mòvile”? Verdi

(89) 12, 14-15 IO29: Lo más alucinante es que podía volar y es como esa canción [símbolo musical] Can belove i can fly [símbolo musical]

En la misma línea, también aumentan significativamente los antropónimos en inglés (que aparecen en hasta un $29 \%$ de las narraciones), de los que mostramos a continuación algunos Ejemplos (90 a 94):

(90) 12, 14-15 IA15: Me llamo Lowis Mackeng [...] La profe Anna estaba pasando lista Maria Hant, Lowis Mackeng...

(91) 12, 14-15 SO28: Un Mathew muy saltón [título]. Al final Mike y Mathew se fueron a sus casas

(92) 12, 14-15 SO29: ¡Thomas! ¡Wilson no está! [...] - ¡Hola Lili! - ¡Buenos dias, Edmun!

(93) 12, 14-15 SA36: - Bueno, pues yo soy Grace, y estarás bien con nosotros. - Gracias, yo soy Margo. [...] Grace apoyó la cara contra Gus

(94) 12, 14-15 SA23: Mi nombre es Phoebe Bug - Yo soy Loreen Wathson 
Las interferencias con el valenciano, por su parte, han aumentado con respecto al grupo de 10 años (aparecen en el $14 \%$ de las narraciones) y de nuevo se dan exclusivamente en los informantes de centros bilingües, con el mismo tipo de ejemplos que ya hemos ido viendo, a los que se añade algún uso que podría interpretarse como generador de humor en los Ejemplos 95 a 97:

（95） 12, 14-15 Bo2: Me pegué una toña

(96) 12, 14-15 MA4: Yo seguía tan destrellatado como había estado siempre.

(97) 12, 14-15 BA9: Las aventuras de Cabolo [...] se le hincho la cabeza y unas palomas le pusieron de nombre cabolo por su cabeza

Un elemento de variación que sin duda ha influido en los resultados de las narraciones humorísticas del grupo de estudiantes de 12 años con respecto a los anteriores es el hecho de que el tema de la redacción fuera distinto. En este caso se trataba de relatar lo que sucedía tras despertarse una mañana habiéndose convertido en un insecto. Aunque de nuevo se incluían indicaciones sobre 'cómo te comunicarías con ellos', lo cierto es que disminuyen significativamente los ejemplos respecto a esta cuestión, probablemente por la percepción de que, aunque los animales se comunican entre ellos, no se trata de lenguas como tales. Del mismo modo, no aparece ninguna mención a asignaturas en las que puedan rastrearse referencias a las lenguas. Así pues, los comentarios sobre otros modos de comunicarse y las actitudes hacia ellos se reducen bastante (apenas se muestran en el 19 $\%$ de las narraciones). Sin embargo, lo que llama la atención es que en la totalidad de estos casos se mantiene, como en el grupo anterior, la percepción de que la comunicación, a pesar de la extrañeza o dificultad inicial, es fácil y divertida (Ejemplos 98 a 101):

(98) 12, 14-15 IO6: Lo que más mola es que podía comunicarme con los insectos.

(99) 12, 14-15 IA11: Encontré un libro que te enseñaba a hablar el idioma abejudo y aprendí a hablarlo ¡me encantaba!

(100) 12, 14-15 IO25: Me acerce hacia el sofa y me encuentro una nota que decía [signos incomprensibles] parecia un idioma muy extranjero pero seguramente sera el de los insectos. Fui hacia el ordenador y saltando en cada tecla de las lteras correspondientes consegui la frase "traductor del idioma de los insectos al español"

(101) 12, 14-15 AA13: ;El me hablo el idioma mosca! Así que me puse a investigar como podia hablar con el y que me entienda y al final... ¡Ajá! Pensé en utilizar el código Morse porque mi abuela y yo lo utilizábamos constantemente. 
Hasta aquí el análisis de resultados según grupos de edad. A continuación, comentamos de manera comparativa los tres grupos para observar la evolución entre ellos respecto a los fenómenos de multicompetencia lingüística.

\subsection{Multicompetencia lingüŕstica en el grupo de 12 años}

Realizando una comparativa de los resultados por franjas de edad, obtenemos los datos que reflejamos a través de la Tabla 1 (con el número de ejemplos encontrados en las narraciones humorísticas)

Tabla 1. Resultados

\begin{tabular}{llccc}
\hline Franja de edad & & 8 años & 10 años & 12 años \\
\hline Cambios de código & Extranjerismos & 3 & 16 & 33 \\
& Antropónimos & 6 & 7 & 25 \\
& Valencianismos & 9 & 9 & 12 \\
\multirow{2}{*}{ Diversidad lingüística } & Lenguas en asignaturas & 7 & 9 & 0 \\
& Comunicación & 33 & 73 & 16 \\
\hline
\end{tabular}

Podemos visualizar mejor estos datos en el Gráfico 5, que los muestra porcentualmente:

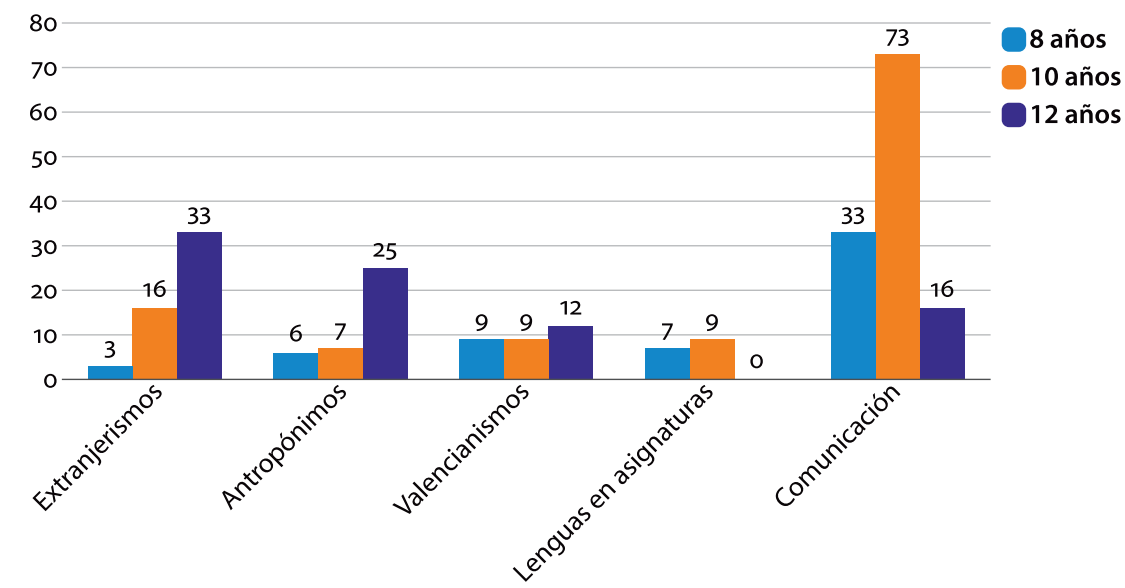

Gráfico 5. Comparativa de resultados por franja de edad

Como podemos observar, en los tres fenómenos de cambio de código (presencia de extranjerismos, antropónimos en otras lenguas e interferencias del 
valenciano) se aprecia un progresivo aumento según se avanza en la edad de los informantes, que podemos explicar por su creciente multicompetencia lingüística. En el caso de los valencianismos es en el que menor diferencia se da; sin embargo, la distancia es bastante más significativa por lo que se refiere a la presencia de términos en otras lenguas y de personajes denominados con nombres extranjeros, que aparecen con mucha mayor frecuencia en el grupo de 12 años. Por el motivo que ya hemos mencionado (el tema distinto para las narraciones en los grupos de 8 y 10 años frente a los de 12) no podemos extraer conclusiones determinantes respecto al hecho de que en el último de ellos aparezcan menos referencias al multilingüismo y los modos de comunicación entre lenguas (o ninguna alusión a otros idiomas al mencionar asignaturas). No obstante, respecto al primero de estos temas, sustancial para entender cómo se percibe el plurilingüismo, sí observamos un visible aumento de las referencias a la diversidad lingüística en el grupo de 10 respecto al de 8. En muchos casos, como hemos tratado de explicar, con connotaciones positivas, además de que tal diversidad es vista como un fenómeno que provoca hilaridad, diversión y, por tanto, humor.

En efecto, con respecto a la conexión con el humor, los comentarios acerca de las diferentes lenguas y los distintos modos de comunicación evidencian que se perciben como algo que genera comicidad y que es motivo de risa en la mayoría de las ocasiones, en los tres grupos de edad. Otro aspecto en común es que no apreciamos voluntad humorística significativa ni en los ejemplos de interferencias del valenciano ni en los extranjerismos, que pueden interpretarse más bien como meramente descriptivos. Sin embargo, sí se observa una voluntad humorística en las denominaciones de personajes con nombres extranjeros en los grupos de 8 y 10 años, algo que desaparece en el de 12, donde su presencia puede explicarse simplemente como una muestra de su competencia en L2 ya adquirida.

\section{Conclusiones}

Cuando abordamos este estudio acerca de la multicompetencia lingüística en el humor infantil, desconocíamos hasta qué punto en las narraciones humorísticas de nuestro corpus encontraríamos fenómenos relacionados de manera directa o indirecta con la competencia plurilingüe de los informantes. A la luz de los resultados que hemos mostrado, y respondiendo a las preguntas de investigación que nos planteábamos, podemos afirmar, respecto a la primera de ellas, que sí se producen cambios de código que muestran multicompetencia lingüística en las narraciones humorísticas infantiles de nuestro corpus. Ello sucede en un porcentaje que va aumentando según lo hace la edad de los informantes; por otro lado, las principales lenguas intercambiadas son, por este orden, el inglés y el

\section{2nd proofs}


valenciano. Estos ejemplos de cambios de código pueden interpretarse, o bien como algo inconsciente y anecdótico, en el caso de los valencianismos, porque se trata mayoritariamente de interferencias involuntarias, fruto del bilingüismo de los informantes, o bien como algo voluntario, en el caso de los extranjerismos (mayoritariamente anglicismos).

Respecto a la pregunta de si podrían interpretarse tales cambios de código como el resultado de una voluntad manifiestamente humorística, consideramos que en su gran mayoría, excepto en contadas excepciones, no tienen como finalidad consciente generar humor, a pesar de la intención humorística del conjunto del texto, sino que, o bien obedecen a la voluntad de describir un contexto extraño, o bien simplemente son un reflejo de la realidad multilingüe experimentada. Donde en cambio sí hemos advertido voluntad de generar humor a través del cambio de código es a través de las denominaciones de los personajes en otras lenguas distintas del español, aunque solo en los grupos de edad de 8 y 10 años.

Por otro lado, en relación con la pregunta de si se hacía referencia en tales narraciones a la diversidad lingüística y el multilingüismo, podemos afirmar claramente que sí, también en una proporción cronológicamente creciente. Las menciones son de índole muy diversa, pero destacamos que los numerosos casos que ejemplifican comunicación en otras lenguas podemos interpretar que tienen un valor pretendidamente humorístico, en la medida en que hablar en otro idioma resulta algo cómico para los informantes (como lo puedan ser los elementos escatológicos, o como se manifiesta en las exageraciones o las interjecciones). En otras ocasiones, por supuesto, los comentarios sobre la diversidad lingüística y la comunicación en varias lenguas podrían obedecer a un elemento de extrañamiento, de constatación de una realidad, pero sin un componente forzosamente humorístico. En cualquier caso, las actitudes hacia otras lenguas distintas de la o las propias que subyacen en los ejemplos son mayoritariamente positivas, además de que reflejan interés, curiosidad y diversión, lo cual es estimulante de cara a los enfoques plurilingües de la política educativa más reciente.

Entre las limitaciones de nuestro estudio podemos mencionar la heterogeneidad de las narraciones y de los fenómenos abordados. Sin embargo, ello nos ha abierto el camino hacia nuevas investigaciones que podrían centrarse bien en una única franja de edad, bien en uno solo de los aspectos estudiados. En cualquier caso, confiamos en que el análisis de la multicompetencia lingüística en las narraciones humorísticas de nuestro corpus haya permitido ahondar de algún modo en las complejas relaciones entre humor y lenguaje.

\section{2nd proofs}




\section{Referencias}

Block, David. 2006. Multilingual Identities in a Global City. London Stories. UK: PalgraveMacMillan. https://doi.org/10.1057/9780230501393

Bronckart, Jean-Paul, y Ecaterina Ulea Bronckart. 2015. “¿Hay algo más natural que el multilingüismo?” En Para una ingeniería didáctica de la educación plurilingüe, ed. por Inés Garcia-Azkoaga, e Itziar Idiazabal, 21-38. Bilbao: UPV/EHU.

Cenoz, Jasone, y Durk Gorter (eds). 2015. Multilingual Education: Between Language Learning and Translanguaging. Cambridge: Cambridge University Press.

Cenoz, Jasone (ed). 2017. "Future directions in adult multilingualism." International Journal of Multilingualism 14:1: 1-5, https://doi.org/10.1080/14790718.2017.1258976

Cook, Vivian. 2012. "Multi-competence." In The Enciclopedia of Applied Linguistics, ed. por Carol A. Chapelle, 3768-3774. New York: Wiley-Blacwell.

Cook, Vivian and Li Wei (eds). 2016. The Cambridge Handbook of Linguistic MultiCompetence. Cambridge: Cambridge University Press. https://doi.org/10.1017/CBO9781107425965

Dewaele, Jean-Marc. 2016. "Multi-competence and emotion." En The Cambridge Handbook of Linguistic Multi-Competence, ed. por Vivian Cook y Li Wei, 461-477. Cambridge: Cambridge University Press. https://doi.org/10.1017/CBO9781107425965.022

Galindo Merino, Ma del Mar. 2017. "La identidad de género a través del humor en niños y niñas de 9-10 años." Círculo de Lingüística Aplicada a la Comunicación (CLAC) 70: 99-118.https://doi.org/10.5209/CLAC.56319

Garcia-Azkoaga, Inés Maa, and Idiazabal, Itziar (eds). 2015. Para una ingeniería didáctica de la educación plurilingüe. Bilbao: UPV/EHU.

Gombert, Jean Émile. 1992. Metalinguistic development. New York: Wheatsheaf.

Lleó, Conxita (ed). 2006. Interfaces in Multilingualism. Amsterdam: John Benjamins. https://doi.org/10.1075/hsm.4

Marimón, Carmen. 2017. "Estrategias para construir humor. Las figuras retóricas en relatos humorísticos de niños de 8 y 12 años." Círculo de Lingüística Aplicada a la Comunicación (CLAC) 70: 61-80.

Martin, Rod A. 2007. The Psychology of Humor: An Integrative Approach. Burlington: Elsevier Academic Press (traducción española de Fernández Escudero, Ma Ángeles. 2008. Madrid: Orión). https://doi.org/10.1016/B978-012372564-6/50024-1

Martín Peris, Ernesto (coord.). 2008. Diccionario de términos clave de ELE. Madrid: SGEL. http://cvc.cervantes.es/ensenanza/biblioteca_ele/diccio_ele/default.htm

Martínez Egido, José Joaquín. 2017. "La expresión de la subjetividad en el humor infantil: elementos valorativos, atenuadores e intensificadores." Círculo de Lingüística Aplicada a la Comunicación (CLAC) 70: 81-98.

Martin-Jones, Marilyn, Adrian Blackledge, y Angela Creese (eds). 2012. The Routledge Handbook of Multilingualism. London/New York: Routledge. https://doi.org/10.4324/9780203154427

Moreno Cabrera, Juan Carlos. 2016. Multilingüismo y lenguas en contacto. Madrid: Síntesis.

Pavlenko, Aneta. 2012. "Multilingualism and emotions." En The Routledge Handbook of Multilingualism, ed. por Marilyn Martin-Jones, Adrian Blackledge, y Angela Creese, 454-469. London/New York: Routledge.

\section{2nd proofs}


Portolés Falomir, Laura. 2015. Multilingualism and very young learners. An analysis of pragmatic awareness and language attitudes. Berlin: Mouton de Gruyter. https://doi.org/10.1515/9781501500022

Ruiz-Gurillo, Leonor. 2012. La lingüística del humor en español. Madrid: Arco/Libros.

Ruiz-Gurillo, Leonor. 2017. "Idiomas inventados en las narraciones humorísticas de niños de 8 a 12 años." Círculo de Lingüística Aplicada a la Comunicación (CLAC) 70: 43-59.

Ruiz de Zarobe, Yolanda, y Leyre Ruiz de Zarobe (eds). 2017. Multilingualism and L2 Acquisition. New perspectives on Current Research. London/New York: Routledge.

Santamaría Pérez, Ma Isabel. 2017. "La expresión del humor infantil a través de la formación de palabras en narraciones escritas." Círculo de Lingüística Aplicada a la Comunicación (CLAC) 70: 21-42.

Schwieter, John (ed.). 2017. Cognitive Control and Consequences of Multilingualism. Amsterdam: John Benjamins. https://doi.org/10.1075/bpa.2

Timofeeva-Timofeev, Larissa. 2014. "El humor verbal en niños de educación primaria: presentación de un estudio." Femenismo/s 24: 195-219. https://doi.org/10.14198/fem.2014.24.09

Timofeeva-Timofeev, Larissa. 2016. "Children Using Phraseology for Humorous Purposes: The Case of 9-to-10-year-olds.” In Metapragmatics of Humor: Current Research Trends, ed. por Leonor Ruiz-Gurillo, 275-300. Amsterdam: John Benjamins, Series IVITRA Research in Linguistics and Literature. https://doi.org/10.1075/ivitra.14.14tim

Timofeeva-Timofeev, Larissa. 2017. "Metapragmática del humor infantil." Círculo de Lingüística Aplicada a la Comunicación (CLAC) 70: 5-19. https://doi.org/10.5209/CLAC.56314

Warriner, Doris S. 2012. "Multilingual Literacies." In The Routledge Handbook of Multilingualism, ed. by Marilyn Martin-Jones, Adrian Blackledge, and Angela Creese, 514-529. London/New York: Routledge.

\title{
Linguistic multicompetence and code-switching in children's humor
}

\begin{abstract}
The phenomena of multilingualism and linguistic multicompetence has been studied from very different perspectives (Martin-Jones, Blackledge and Creese 2012; Cook and Wei 2016). The objective of this article is to relate them with code-switching and language diversity perceptions in order to study their effect in a corpus (obtained from the I+D project La formación de la conciencia figurativa en la etapa de formación primaria: el humor y la fraseología -FFI2016-76047-P) of humorous stories of children aged 8 to 12. The quantitative and qualitative analysis of the corpus has shown the extent to which implicit and explicit knowledge of several languages at these ages affects the code-switching and the reference to other languages, and whether or not it appears in a way that is intended to be comical (Timofeeva-Timofeev 2017).
\end{abstract}

Keywords: linguistic multicompetence, code-switching, children's humor

\section{2nd proofs}




\section{Dirección para la correspondencia}

Susana Pastor Cesteros

Facultad de Filosofía y Letras

Dpto. Filología Española, Lingüística General y Teoría de la Literatura

Universidad de Alicante

03690 Alicante

España

SPC@ua.es

\section{Notas biográficas}

Susana Pastor Cesteros es profesora titular de Lingüística de la Universidad de Alicante, especializada en Lingüística aplicada y Español como Lengua Extranjera. Fundadora y directora actual del grupo de investigación ACQUA (Adquisición y enseñanza de segundas lenguas y lenguas extranjeras de la UA). Presidenta de la Asociación para la Enseñanza del Español como Lengua Extranjera, ASELE (2008-2016). Evaluadora externa del Programa de Español de la University of Hong Kong (2015-2018). Ha participado en diversos proyectos de investigación y colabora en calidad de evaluadora externa de revistas científicas de su especialidad. Sus publicaciones pueden consultarse en el portal de ACQUA. 